\title{
Studies on the influence of structurally different peroxides in polypropylene/ethylene alpha olefin thermoplastic vulcanizates (TPVs)
}

\author{
R. R. Babu, N. K. Singha, K. Naskar* \\ Rubber Technology Centre, Indian Institute of Technology, Kharagpur-721302, West Bengal, India \\ Received 21 January 2008; accepted in revised form 15 February 2008
}

\begin{abstract}
Novel thermoplastic vulcanizates (TPVs) based on polypropylene (PP) and new generation ethylene-octene copolymer (EOC) have been developed by dynamic vulcanization process, which involves melt-mixing and simultaneously crosslinking a rubber with a thermoplastic. In this paper technologically compatibilized blends of PP and EOC were dynamically vulcanized by coagent assisted peroxide crosslinking system. The effect of structurally different types of peroxides, namely dicumyl peroxide, di-tert butyl peroxy isopropyl benzene and tert-butyl cumyl peroxide with varying concentrations on the properties on TPVs was mainly studied. The physico-mechanical, thermal and morphological properties of these TPVs were characterized by using X-ray diffraction (XRD), differential scanning calorimeter (DSC) and scanning electron microscopy (SEM).
\end{abstract}

Keywords: polymer blends and alloys, polypropylene, ethylene octene copolymer, thermoplastic vulcanizate, peroxide

\section{Introduction}

Thermoplastic Elastomers (TPEs) have the processing characteristics of a thermoplastic and functional performance of a conventional thermoset rubber. TPEs are gaining importance day-by-day because of their wide range of applications especially in the automotive industries [1]. TPEs can be broadly classified into two groups: block copolymers and rubber-plastic blends. Blending of two or more polymers can give rise to better balance of properties than that of individual components. Polyolefin based blends have attracted much attention due to easy processability and broad spectrum of properties which can also be obtained at competitive price. Dynamic vulcanization is the process of mixing a thermoplastic and a rubber, and the latter is being cross-linked under dynamic conditions. The process needs to be carried out at high shear rate above the melting temperature of thermoplastic and also at sufficiently high temperature to activate and to complete the process of vulcanization. The material prepared, so called thermoplastic vulcanizate (TPVs) or dynamic vulcanizate is characterized by processing behavior like thermoplastic at elevated temperature and performance properties of vulcanized rubber at ambient temperature [2]. In this dynamic vulcanization, small cross-linked rubber particles are dispersed in the uncross-linked thermoplastic polymer matrix as microgels, although the content of rubber exceeds the thermoplastic content in most cases. Dynamic vulcanization is a route to configure thermoplastic elastomer, which have better properties than those of conventional block co-polymer TPEs. Gessler was the first to claim dynamically vulcanized blends in 1962 [3]. Significant improvement in the field of TPVs

*Corresponding author, e-mail: knaskar@rtc.iitkgp.ernet.in (C) BME-PT and GTE 
was achieved by Coran and Patel [4] by fully vulcanizing the rubbery phase without affecting the thermoplasticity of the blends. Further development in this field was advanced by Abdou-Sabet and Fath [5] by using phenolic resin as curative in polypropylene/ ethylene propylene diene rubber (PP/EPDM) which not only crosslink rubbery phase but also involve in compatibilizing the two phases by forming graft-links between the blend components. Though several cross-linking agents have been employed to cross-link the elastomer phase in TPVs, the phenolic resin, peroxide and silane cross-linking systems have gained considerable commercial importance. Major advantages of using peroxide cross-linking system are that they have improved high temperature resistance, reduced compression set, their ability to vulcanize both saturated and unsaturated rubbers and reversion resistance [6]. Besides the advantages of peroxide, there are also some disadvantages like radical transfer, polymer scission, dehydrohalogenation (in halogenated polymers like polyvinyl chloride, chlorinated polyethylene etc.) and oxygenation etc.
These types of competing reactions can be partially overcome by employing suitable coagent. Coagents are basically multifunctional reactive monomers mostly containing terminal unsaturation, which undergo addition reaction to stabilize the highly reactive polymer radicals. Principally it suppresses the chain scission reaction and enhances the extent of cross-linking. General mechanism for coagent assisted peroxide crosslinking along with possible competing reactions is shown in Figure 1 [7]. Naskar and Noordermeer [8,9] investigated the possible use of peroxides in the production of PP/EPDM TPVs. They showed the influence of structurally different peroxides with special reference to the mechanical properties. Multifunctional peroxide [10], having peroxide and coagent functionality in a single molecule was also explored to overcome the unpleasant smell and blooming characteristics produced by decomposed products of some peroxides. Thitithammawong et al. [11] studied the influences of different peroxides in PP/epoxidized natural rubber (ENR) TPVs. Chatterjee and Naskar [12] also studied the properties of

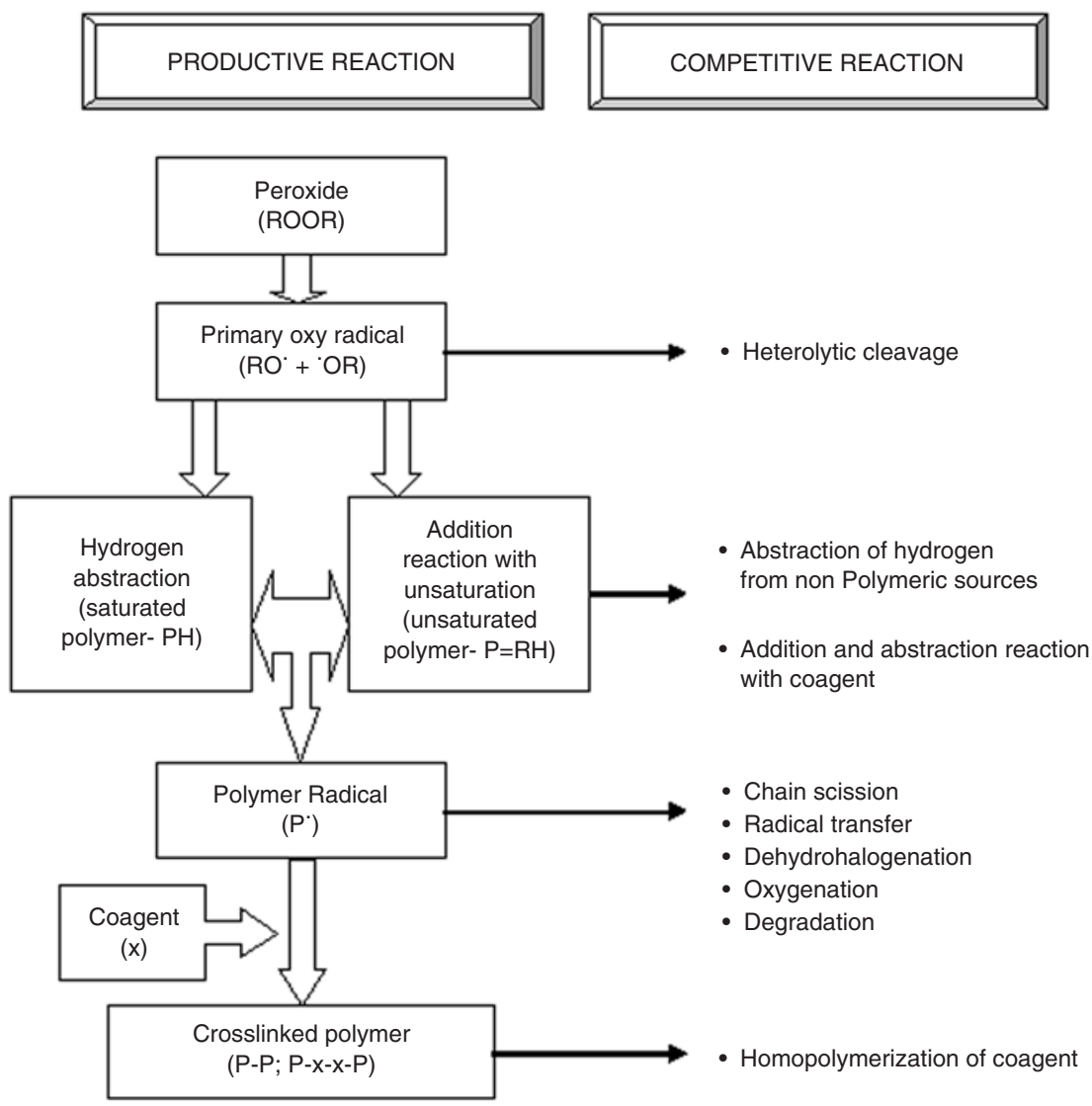

Figure 1. Various productive and competitive reactions involved in coagent assisted peroxide system 
dicumyl peroxide cured TPVs based on maleated ethylene propylene rubber (m-EPM) and PP using maleated-PP as compatibilizer.

Interrelationships of morphology, properties and processing characteristics of dynamically vulcanized blends have been reviewed by Karger-Kocsis [13]. Properties of the TPVs depend on their morphology and the crosslink density in the elastomer phase. An investigation on the correlation between mechanical and morphological properties of oil extended PP/EPDM TPVs was carried out by Sengupta [14]. Recently Naskar [15] made an extensive review on structure-property relationship of PP/EPDM TPVs. Considerable researches have been pursued on PP/EPDM TPVs, but TPVs based on ethylene octene copolymer and PP were not well explored. Ethylene octene copolymers (EOC) or plastomers are a relatively new class of polymers that emerged with recent advances in metallocene polymerization catalysts. Representing one of the fastest growing synthetic polymers, EOCs are compatible with most of the olefinic materials which impart excellent impact strength for semicrystalline polymers, and offer unique performance capabilities for compounded products [16]. EOC is gradually replacing the field of applications of EPDM rubber and can be considered as a suitable alternative for EPDM. Addition of peroxide in PP/EOC blend involves two major competing reactions:
EOC cross-linking and PP degradation by $\beta$-scission. Final product properties are thus dependent on the balance among those two competing reactions. The primary objective of the present investigation is to study the influence of structurally different peroxides on the physico-mechanical, thermal and morphological characteristics of the dynamically cross-linked polypropylene (PP) and ethylene octene copolymer (EOC) blends at a fixed blend ratio.

\section{Experimental}

\subsection{Materials}

The general purpose polyolefin elastomer Exact 5171 (specific gravity, $0.870 \mathrm{~g} / \mathrm{cc}$ at $23^{\circ} \mathrm{C}$; comonomer (octene) content $13 \%$; melt flow index (MFI), $1.0 \mathrm{dg} / \mathrm{min}$ at $190^{\circ} \mathrm{C} / 2.16 \mathrm{~kg}$ ), was commercialized by Exxon Mobil Chemical Company, USA. Polypropylene (PP) (Specific gravity, $0.9 \mathrm{~g} / \mathrm{cc}$ at $23^{\circ} \mathrm{C}$; melt flow index, $3.0 \mathrm{~g} / 10 \mathrm{~min}$ at $230^{\circ} \mathrm{C} / 2.16 \mathrm{~kg}$ ) was obtained from IPCL, India. The coagent, tri allyl cyanurate (SR 507A; Specific gravity, $1.12 \mathrm{~g} / \mathrm{cc}$ at $23^{\circ} \mathrm{C}$ ) was obtained from Sartomer Company, USA. Three different types of peroxides were used and their commercial names, chemical names and half life data were given in the Table 1. These materials were obtained from Akzo Nobel Chemical Company, The Netherlands.

Table 1. Commercial names, chemical names, structures and half-life $\left(t_{1 / 2}\right)$ data of various peroxides used

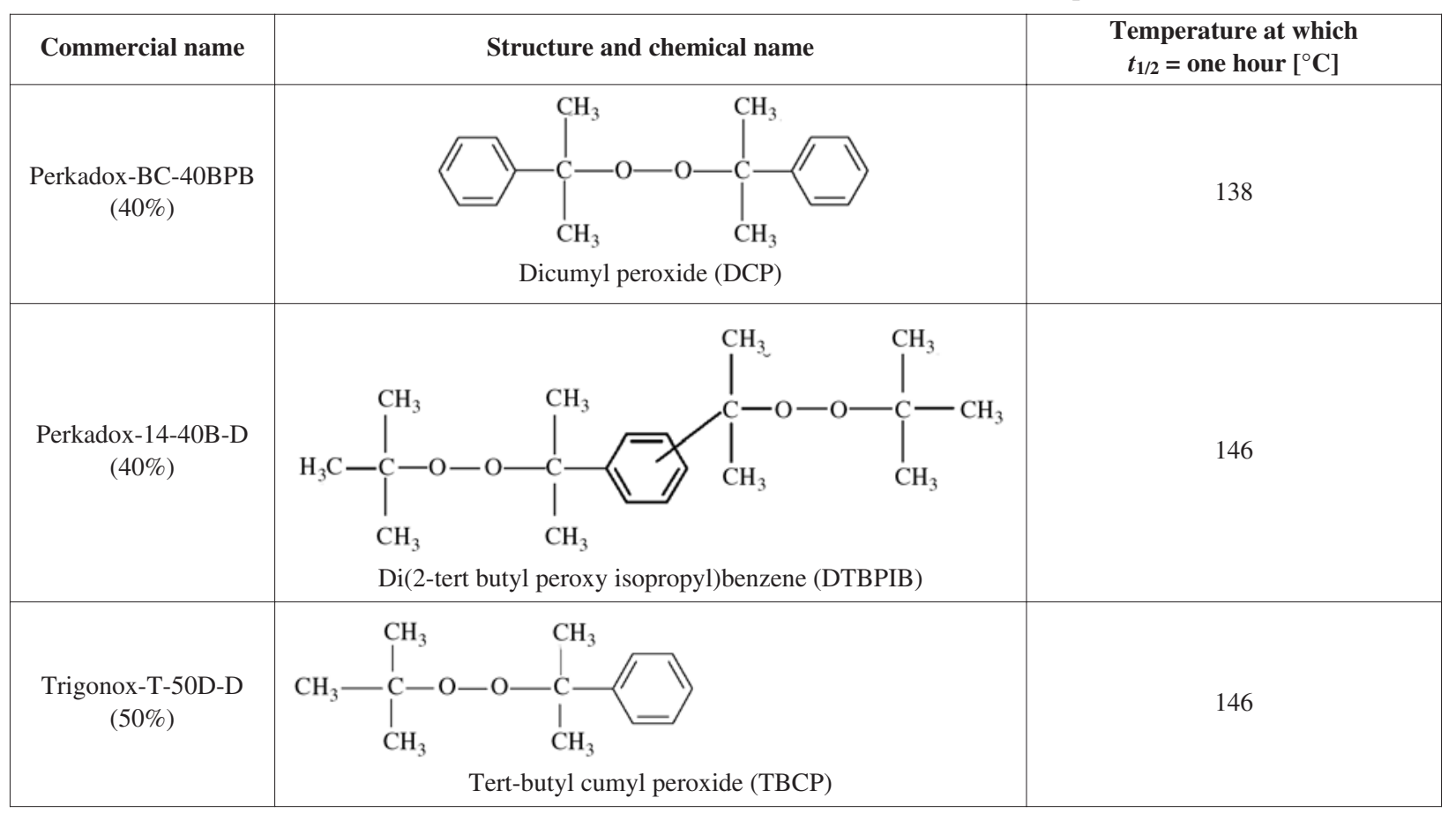




\subsection{Preparation of PP/EOC TPVs}

Compositions showing blend constituents, type and concentration of different peroxides are shown in the Table 2. The experimental variables are the type and concentration of peroxides. All TPVs were prepared by a batch process in a Haake Rheomix600 internal mixer, having a mixing chamber volume of $85 \mathrm{~cm}^{3}$ with a rotor speed of $80 \mathrm{rpm}$ at $190^{\circ} \mathrm{C}$. Immediately after mixing, the composition was passed once through a cold tworoll mill to achieve a sheet of about $2 \mathrm{~mm}$ thickness. The sheet was cut and pressed in a compression molding machine (Moore Press, Birmingham, $\mathrm{UK})$ at $200^{\circ} \mathrm{C}, 4 \mathrm{~min}$ and $5 \mathrm{MPa}$ pressure. The sheet was then cooled down to room temperature under pressure. Test specimens were die-cut from the compression molded sheet and used for testing after $24 \mathrm{hrs}$ of storage at room temperature. Since different peroxides not only differ in molecular weight but they also have different relative functionality depending on the number of peroxy groups present. From the structures of different peroxides (Table 1), it is clear that one mole of DTBPIB contains two peroxide groups where as DCP and TBCP contain one peroxide group. The technical definition of an equivalent is the amount of substance it takes to combine with one mole of hydrogen ion. A milliequivalent (meq) is $1 / 1000$ of an equivalent, for a monovalent ion one milliequvalent is equal to one mole. In practice, the amount of substance in equivalent often has a small magnitude, so it is frequently expressed in terms of milliequivalents. In order to compare different peroxides, concentrations employed were expressed in terms of milliequivalents per $100 \mathrm{~g}$ of pure EOC. TPVs prepared by different peroxides are designated as D for dicumyl peroxide (DCP), T for tertbutyl cumyl peroxide (TBCP) and DT for di(tertbutylperoxyisopropyl)benzene (DTBPIB). The number in the compound designation (Table 2) corresponds to the respective concentration of peroxide in milliequivalents.

\subsection{Testing procedure}

Tensile tests were carried out according to ASTM D412-98 on dumb-bell shaped specimens using a universal tensile testing machine Hounsfield H10KS (UK) at a constant cross-head speed of $500 \mathrm{~mm} / \mathrm{min}$. Tear strength was carried out according to ASTM D-624-81 test method using unnicked $90^{\circ}$ angle test piece. Tension set was performed at room temperature with a stretched condition for 10 minutes at $100 \%$ elongation according to ASTM D412-98 method. A Rubber Process Analyzer, RPA-2000 (Alpha Technologies, USA), was used to study the cure-characteristics of the different types of peroxides employed only on the EOC part (PP omitted) at $180^{\circ} \mathrm{C}$ for 30 minutes at $2.79 \%$ strain and a frequency of $1 \mathrm{~Hz}$. Equilibrium solvent swelling measurement was carried out on the pure EOC gum vulcanizate to determine the crosslink density of the EOC. The crosslink density was calculated using the modified Flory-Rehner equation. A circular test piece of $2 \mathrm{~mm}$ thickness was allowed to swell in cyclohexane for about 48 hours to attain equilibrium swelling condition. Initial weight, swollen weight and deswollen or dried weight were taken and substituted in the equation to get the crosslink density values.

Wide angle X-ray Diffraction was performed with a Philips PW 1840 X-ray diffractometer (The Netherlands) with a copper target $\left(\mathrm{CuK}_{\alpha}\right)$. The samples were scanned from 10 to $40^{\circ}(2 \theta)$ at a scanning speed of $0.05^{\circ} 2 \theta / \mathrm{s}$ with an operating voltage of $40 \mathrm{kV}$ and current of $20 \mathrm{~mA}$. Differential scanning calorimeter (DSC) measurements were carried out using a DSC Q100 V.8.1 (TA instruments,

Table 2. TPV compositions in phr (parts per hundred rubber)

\begin{tabular}{|l|c|c|c|c|c|c|c|c|c|c|c|c|c|}
\hline COMP-ID & PP-EOC & D1 & D3 & D5 & D7 & T1 & T3 & T5 & T7 & DT1 & DT3 & DT5 & DT7 \\
\hline EOC & 100 & 100 & 100 & 100 & 100 & 100 & 100 & 100 & 100 & 100 & 100 & 100 & 100 \\
\hline PP & 50 & 50 & 50 & 50 & 50 & 50 & 50 & 50 & 50 & 50 & 50 & 50 & 50 \\
\hline DCP & - & $0.68(1)$ & $2.03(3)$ & $3.38(5)$ & $4.73(7)$ & - & - & - & - & - & - & - & - \\
\hline TBCP & - & - & - & - & - & $0.42(1)$ & $1.26(3)$ & $2.10(5)$ & $2.94(7)$ & - & - & - & - \\
\hline DTBPIB & - & - & - & - & - & - & - & - & - & $0.43(1)$ & $1.27(3)$ & $2.13(5)$ & $2.98(7)$ \\
\hline TAC & - & 2 & 2 & 2 & 2 & 2 & 2 & 2 & 2 & 2 & 2 & 2 & 2 \\
\hline
\end{tabular}

Values in the parenthesis corresponds to the concentration of corresponding peroxide expressed in milliequivalent.

D - Dicumyl peroxide (DCP)

T - Tertbutylcumyl peroxide (TBCP)

DT - Di(tertbutylperoxyisopropyl) benzene (DTBPIB) 
USA). The scans were taken in the temperature range from -80 to $200^{\circ} \mathrm{C}$ with a programmed heating rate of $10^{\circ} \mathrm{C} / \mathrm{min}$ under nitrogen atmosphere. Phase morphology of the cryo-fractured samples of various blends was investigated by Digital Scanning Electron Microscope (SEM) (JEOL JSM 5800, Japan).

\section{Results and discussion}

\subsection{Mechanical properties}

Mechanical properties are considerably improved by the dynamic vulcanization process. Influences of different peroxides on the mechanical properties of the PP/EOC TPVs are shown in the Figures 2-6. Effect of peroxide on a particular system depends on the nature of the polymer, type and concentration of peroxide and also upon the reactivity of other components in the system. It is generally accepted that peroxide causes cross-linking in EOC and also causes $\beta$ chain scission in PP by abstraction of tertiary hydrogen atom from the main chain polymers. Addition of co-agents, which are highly reactive towards free radicals, can improve the cross-linking tendency to form tighter network in EOC and also decrease the extent of degradation in PP. Figure 2 shows the improvement in tensile strength with the addition of co-agent assisted different peroxide system with varying concentration. It is observed that tensile strength increases initially, reaches a maximum and then decreases for DCP and DTPBIB, whereas for TBCP after initial increase it levels off. DCP cured TPVs show maxi-

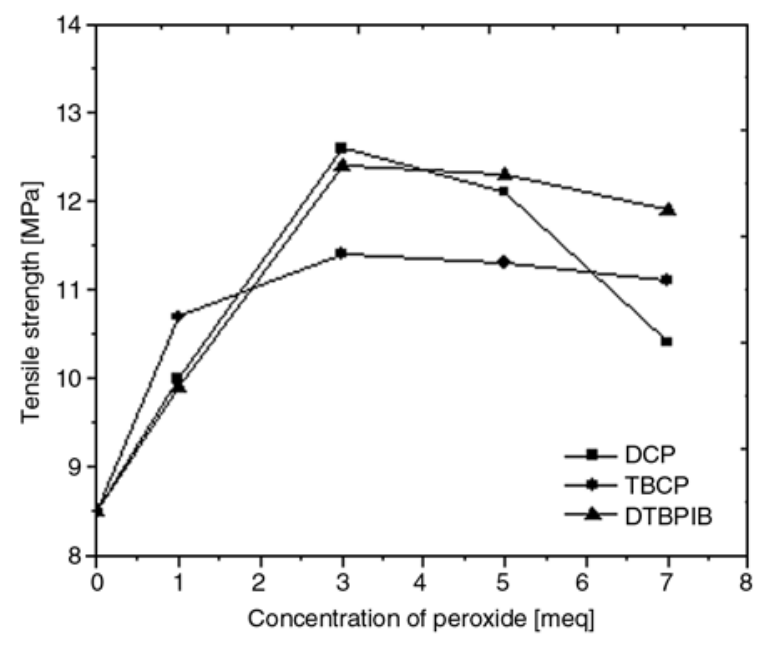

Figure 2. Tensile strength as a function of concentration of different peroxides. (Composition corresponds to Table 2)

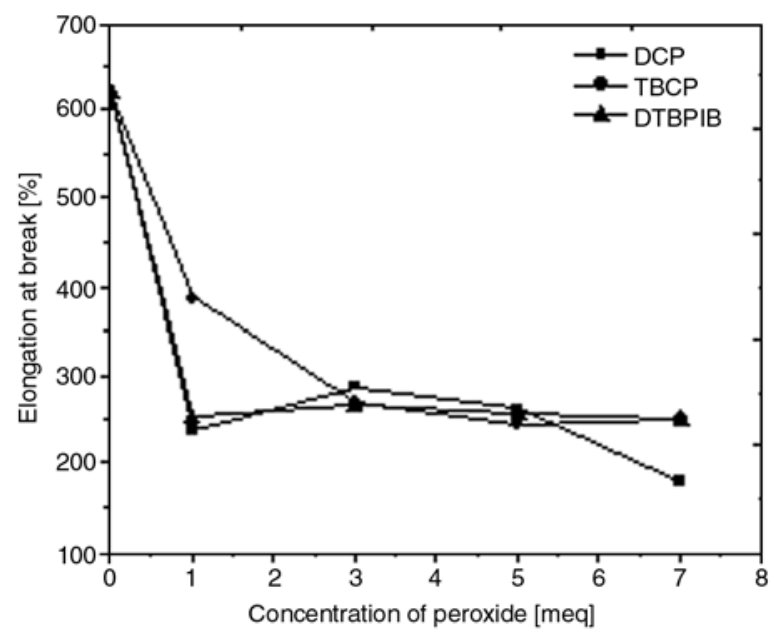

Figure 3. Elongation at break as a function of concentration of different peroxides. (Composition corresponds to Table 2)

mum tensile strength at 3 meq concentration. On decomposition, DCP and DTBPIB produce highly reactive radicals and the amount of generated radicals are also higher than for TBCP [8]. The decrease in tensile strength at higher concentration of DCP and DTBPIB may be due to the severe degradation on the PP phase. Figure 3 shows the elongation at break behavior as a function of concentration of different peroxides. Elongation at break continuously decreases although little difference between the various peroxides used is noticed; TBCP shows a relatively higher value compared to others. Decreasing trend may be associated with the phase inversion on morphology and cross-linking in the EOC phase. Also at higher dosage level, the nature of failure of the samples shows brittle type. Moduli at $100 \%$ elongation as a function of concentration of different peroxides are shown in Figure 4. Modulus value increases initially and then reaches a plateau. DCP shows the highest modulus and TBCP shows the lowest modulus, which corresponds to the trend in tensile strength values. It is observed that crosslinking efficiency of DCP and DTBPIB is higher than TBCP, which is further manifested from the cure characteristics.

Tear strength of the TPVs increase considerably by the dynamic vulcanization process. Influence of different peroxides on the tear strength is shown in Figure 5. During dynamic vulcanization, along with the cross-linking process on EOC phase, grafting of EOC on PP can also take place; the latter improves the interfacial adhesion. DTBPIB shows 


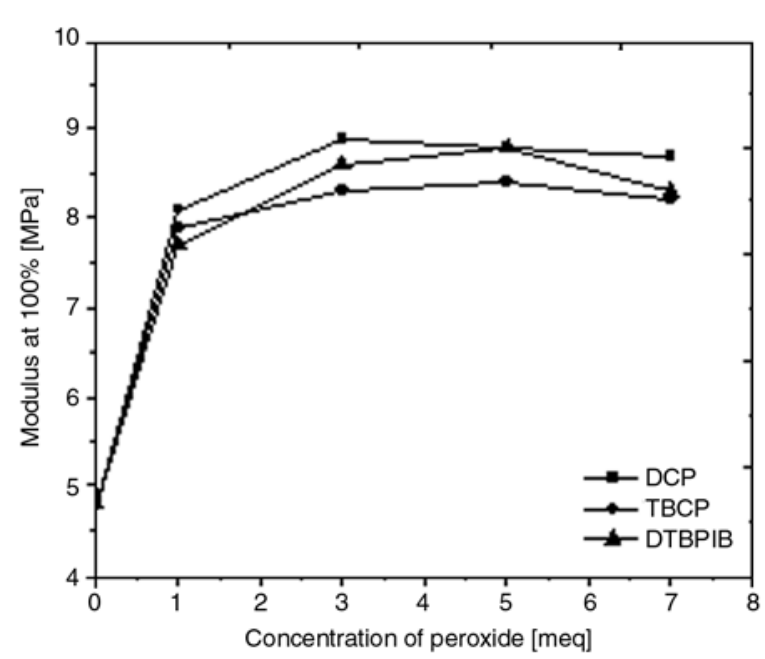

Figure 4. Modulus at $100 \%$ as a function of concentration of different peroxides. (Composition corresponds to Table 2)

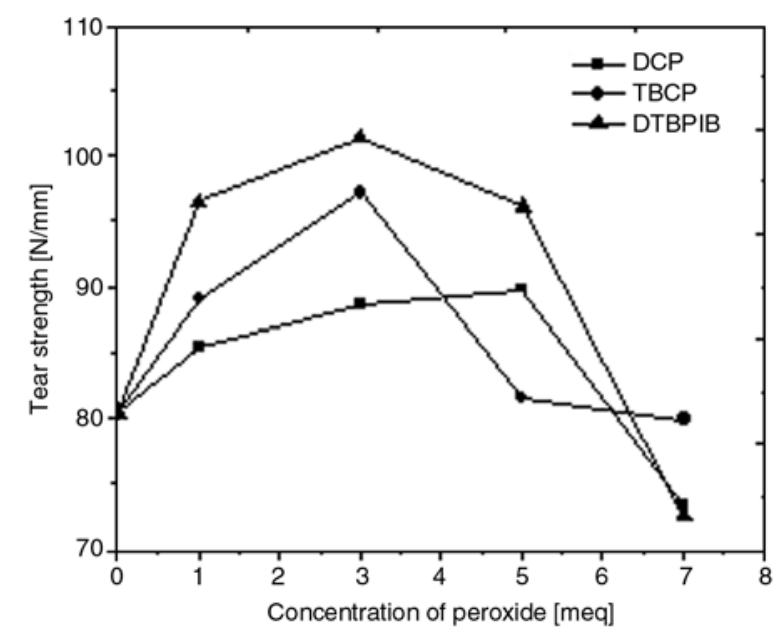

Figure 5. Tear strength as a function of concentration of different peroxides. (Composition corresponds to Table 2)

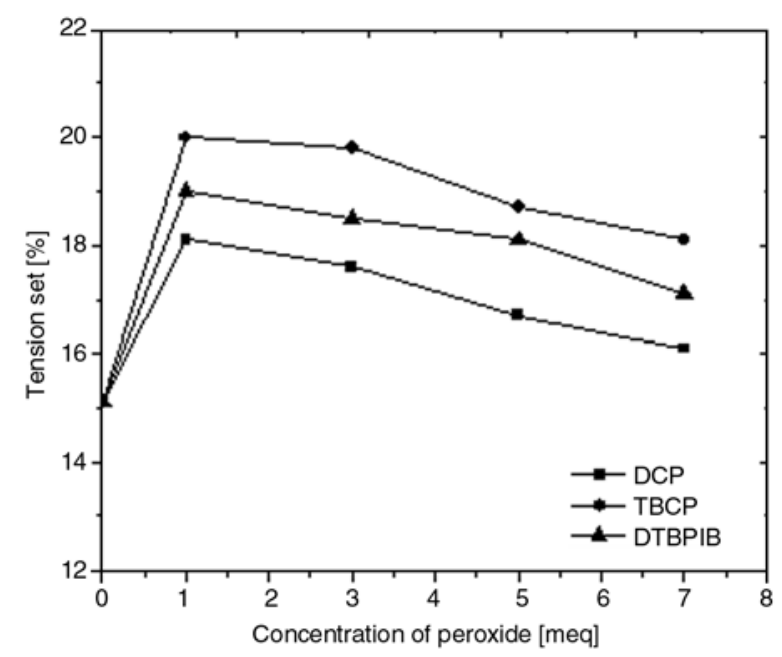

Figure 6. Tension set as a function of concentration of different peroxides. (Composition corresponds to Table 2) the maximum tear strength at 3 meq concentration and DCP shows the least value. Decreasing trend on higher loading of peroxide may be due to severe degradation in PP and thus it has limited extensibility of cross-linked EOC [17]. DTBPIB is expected to form better interfacial cross-linking. Tensions set values as the function of concentration of different peroxides are shown in Figure 6. Unvulcanized blends show lower set values; whereas dynamically vulcanized ones show higher values. However, with further addition of peroxide tension set decreases continuously. This behavior may be explained on the basis of morphological aspect. In unvulcanized blend EOC being the major proportion give rise to continuous phase and PP as dispersed phase. It has been previously reported that EOC exhibit higher viscosity and elastic property than PP [18]. However, phase inversion occurs due to dynamic vulcanization which causes crosslinked EOC as dispersed phase and PP as continuous matrix which is responsible for the higher set values. Further as the concentration of peroxide increases, formation of tighter network causes gradual decrease in set values (higher elastic recovery property). Among the peroxides taken under investigation, DCP exhibits lower set values than DTBPIB and TBCP.

Hence mechanical properties of PP/EOC TPVs by coagent assisted peroxide would be ascribed by the extent of cross-linking in the EOC phase as well as extent of PP degradation. Different types of peroxides have different cross-linking efficiency and exhibit different decomposition pattern under given condition [8] which is responsible for governing final TPV properties.

\subsection{Cure characteristics and crosslink density}

In order to interpret the effect of different peroxides on the PP/EOC TPVs, it is necessary to understand the reactivity and efficiency of different peroxide only on EOC phase (PP omitted). Table 3 shows the cure characteristics for pure EOC gum vulcanizate. It is observed that irrespective of type of peroxide used, increase in concentration of peroxide causes increase in maximum torque (Max $S$ ) and hence delta torque values $(\Delta S)$. This is evident that increase in concentration of peroxide, more radicals are formed and thus higher the crosslinking effi- 
Table 3. RPA test results and crosslink density for pure EOC gum vulcanisates

\begin{tabular}{|l|c|c|c|c|c|c|c|}
\hline $\begin{array}{c}\text { Compound } \\
\text { name }\end{array}$ & $\begin{array}{c}\text { Min S } \\
{[\mathbf{d N m}]}\end{array}$ & $\begin{array}{c}\mathbf{M a x} \mathbf{S} \\
{[\mathbf{d N m}]}\end{array}$ & $\begin{array}{c}\mathbf{\Delta} \mathbf{S} \\
{[\mathbf{d N m}]}\end{array}$ & $\begin{array}{c}\mathbf{T}_{\mathbf{1 0}} \\
{[\mathbf{m}: \mathbf{s}]^{\mathbf{a}}}\end{array}$ & $\begin{array}{c}\mathbf{T}_{\mathbf{9 0}} \\
{[\mathbf{m}: \mathbf{s}]^{\mathbf{a}}}\end{array}$ & $\begin{array}{c}\text { Cure rate } \\
{[\mathbf{\%}]}\end{array}$ & $\begin{array}{c}\text { Crosslink density (v) } \\
\mathbf{1 0}^{-\mathbf{4}} \mathbf{m o l} \mathbf{m} \mathbf{\mathbf { m }}\end{array}$ \\
\hline RD1 & 0.35 & 3.59 & 3.23 & 0.4 & 2.6 & 44.0 & 1.85 \\
\hline RD3 & 0.47 & 4.20 & 3.73 & 0.3 & 2.4 & 48.0 & 2.50 \\
\hline RD5 & 0.87 & 5.06 & 4.19 & 0.3 & 2.3 & 48.0 & 2.88 \\
\hline RT1 & 0.10 & 3.12 & 3.01 & 1.0 & 5.2 & 23.8 & 1.02 \\
\hline RT3 & 0.17 & 4.18 & 4.01 & 0.5 & 4.5 & 24.8 & 1.88 \\
\hline RT5 & 0.27 & 4.40 & 4.12 & 0.4 & 4.2 & 26.1 & 1.96 \\
\hline RDT1 & 0.20 & 3.65 & 3.43 & 0.5 & 5.6 & 19.4 & 1.93 \\
\hline RDT3 & 0.22 & 4.34 & 4.12 & 0.4 & 5.2 & 21.0 & 2.52 \\
\hline RDT5 & 0.43 & 5.20 & 4.77 & 0.3 & 4.2 & 25.9 & 2.69 \\
\hline
\end{tabular}

${ }^{\mathrm{a}} \mathrm{m}: \mathrm{s}-$ minutes:seconds

$\mathrm{R}$ denotes the compound composition in the Table 2 without PP content

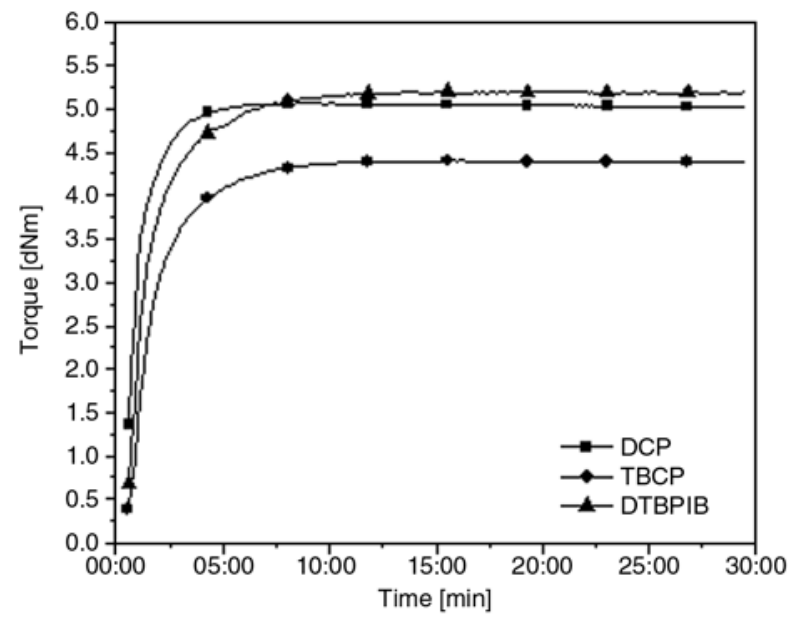

Figure 7. Rheographs for EOC compounds with 5 meq concentration of different peroxides

ciency and simultaneous decrease in scorch $\left(T_{10}\right)$ and optimum cure time $\left(T_{90}\right)$. Figure 7 shows the rheographs of different peroxides on the EOC phase at a concentration of 5 meq. As can be observed from the Figure 7 and Table 3, DTBPIB shows the maximum torque values followed by DCP and TBCP whereas considering the rate of cross-linking i.e., cure rate index (CRI), DCP is the fastest followed by TBCP and DTBPIB.

Crosslink density of pure EOC gum vulcanizate $(v)$ was calculated by using Flory-Rehner equation (Equation (1)):

$\mathrm{v}=-\frac{1}{V_{s}} \cdot \frac{\ln \left(1-V_{r}\right)+V_{r}+\chi\left(V_{r}\right)^{2}}{\left(V_{r}\right)^{1 / 3}-0.5 V_{r}}$

where $V_{s}-$ molar volume of cyclohexane $\left[\mathrm{cm}^{3} / \mathrm{mol}\right]$, $\chi$ - polymer swelling agent interaction parameter, which in this case is 0.306 [19] and $V_{r}$-volume fraction of ethylene-octene copolymer in the swollen network $\left[\mathrm{cm}^{3} / \mathrm{mol}\right]$, which can be expressed by Equation (2):

$$
V_{r}=\frac{1}{A_{r}+1}
$$

where $A_{r}$ is the ratio of the volume of absorbed cyclohexane to that of ethylene-octene copolymer after swelling.

TBCP is found to give lowest crosslink densities, where as DCP and DTBPIB show higher and the latter shows only marginal difference in the values. There was a clear trend of variation in modulus values of TPVs for different peroxide with the delta torque and crosslink density values. Change in elongation at break and tension set values of TPVs with increase in concentration of different peroxide are also well correlated with the extent of crosslink density.

\subsection{Differential scanning calorimeter (DSC)}

DSC data of PP/EOC simple blends along with three different peroxide cured PP/EOC TPVs are shown in the Table 4. The blend shows two glass transitions $\left(T_{g}\right)$ and melting endotherms $\left(T_{m}\right)$. These clearly indicate that the blend components are thermodynamically immiscible. By dynamic vulcanization they are made technologically compatible which is manifested by improvement in mechanical properties. With the addition of peroxide, two major reactions take place. First, glass transition of EOC is shifted to higher temperature which may be due to cross-linking in EOC phase. Secondly, melting endotherm of both EOC and PP phases were diffused or obscured. This can be 
Table 4. DSC data of PP/EOC TPVs with different peroxides

\begin{tabular}{|l|c|c|c|c|c|c|c|c|}
\hline $\begin{array}{c}\text { Compound } \\
\text { name }\end{array}$ & $\begin{array}{c}\mathbf{T g} \\
\mathbf{E O C} \\
{\left[{ }^{\circ} \mathbf{C}\right]}\end{array}$ & $\begin{array}{c}\Delta \mathbf{H}_{\mathbf{f}} \\
\mathbf{E O C} \\
{[\mathbf{J} / \mathbf{g}]}\end{array}$ & $\begin{array}{c}\mathbf{\%} \\
\mathbf{C R Y} \\
\mathbf{E O C}\end{array}$ & $\begin{array}{c}\mathbf{\mathbf { T } _ { \mathbf { m } }} \\
\mathbf{E O C} \\
{\left[{ }^{\circ} \mathbf{C}\right]}\end{array}$ & $\begin{array}{c}\mathbf{T}_{\mathbf{g}} \\
\mathbf{P P} \\
{\left[{ }^{\circ} \mathbf{C}\right]}\end{array}$ & $\begin{array}{c}\Delta \mathbf{H f} \\
\mathbf{P P} \\
{[\mathbf{J} / \mathbf{g}]}\end{array}$ & $\begin{array}{c}\mathbf{\%} \\
\mathbf{C R Y} \\
\mathbf{P P}\end{array}$ & $\begin{array}{c}\mathbf{T}_{\mathbf{m}} \\
\mathbf{P P}\end{array}$ \\
\hline PP & - & - & - & - & 9 & 98.88 & 47.76 & 165 \\
\hline EOC & -51 & 23.34 & 8.05 & 50 & - & - & - & - \\
\hline PP-EOC & -51 & 16.22 & 8.47 & 50 & 7 & 32.75 & 47.50 & 165 \\
\hline D3 & -47 & 15.97 & 8.26 & 52 & 8 & 30.82 & 44.70 & 163 \\
\hline T3 & -48 & 15.51 & 8.02 & 51 & 7 & 31.58 & 45.80 & 164 \\
\hline DT3 & -47 & 15.72 & 8.13 & 52 & 8 & 30.48 & 44.20 & 163 \\
\hline D15 & -45 & 11.32 & 5.86 & 50 & 1 & 23.65 & 34.62 & 150 \\
\hline
\end{tabular}

*DCP of 15 meq concentration

explained on the basis of cross-linking in EOC part and grafting of EOC on PP or grafting of PP on EOC that limits the crystallizing tendency thereby reducing the heat of fusion $\left(\Delta H_{f}\right)$ and percentage crystallinity (\% CRY) of PP as shown in Table 4. DCP and DTBPIB show higher cross-linking efficiency over TBCP and correspondingly affect more the crystallization of PP.

Further to understand the influence of higher dosage level of peroxide, DCP of 15 milliequivalents (meq) concentration was taken for the preparation of TPVs (in Table 4 designated as D15). It is interesting to note that apart from above mentioned effects, perfectness of the crystals in PP (size of the crystals) is affected considerably which is observed by a drastic fall in melting temperature from 165 to $150^{\circ} \mathrm{C}$; glass transition temperature of $\mathrm{PP}$ also decreases from 8 to $1^{\circ} \mathrm{C}$.

\subsection{Wide angle $X$-ray diffraction (WAXD)}

Crystallinity level and pattern of the blends were studied by using wide angle $\mathrm{x}$-ray scattering technique. Figure 8 shows the WAXD pattern of the pure PP, PP-EOC unvulcanized blend and dynamically vulcanized blends. The diffraction pattern has a broad amorphous background superimposed on sharp crystalline peaks. Generally PP has three crystalline structure $\alpha$ (monoclinic), $\beta$ (pseudo hexagonal) and $\gamma$ (triclinic). The diffraction pattern of PP has five sharp diffraction lines corresponding to $110,040,130,111$ and overlapping of 131 with 041 planes in the crystal which are located at a scattering angle $2 \theta$ of $14.2,17.1,18.7,21.4$ and $22.0^{\circ}$ respectively. The pattern shows only $\alpha$ form because there is no line at $2 \theta$ of $16.1^{\circ}$ generally associated with $\beta$ form [20]. Crystallinity pattern can be inferred from the change in position of

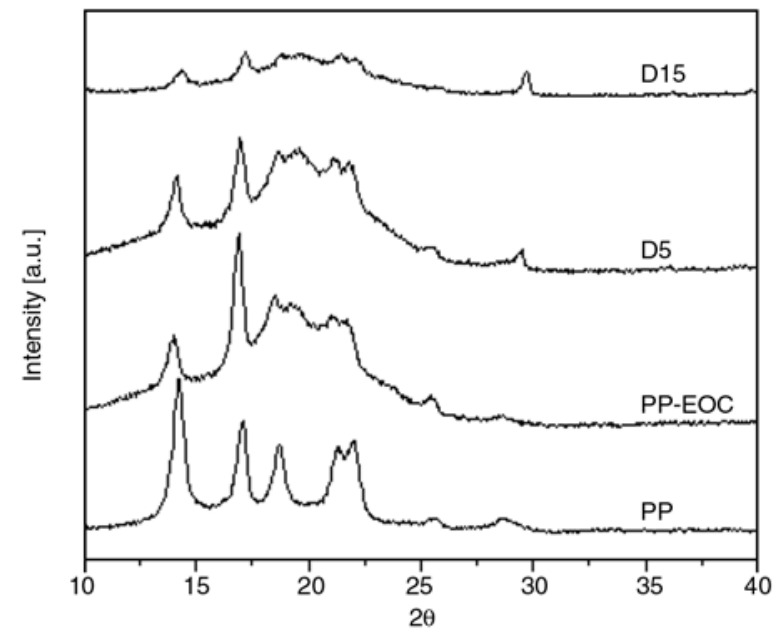

Figure 8. WAXD of PP, unvulcanized and dynamically vulcanized PP/EOC blends

peaks, whereas crystallinity level from the intensity of the peaks. Percentage crystallinity value can be calculated using Equation (3) from the area under the crystalline and amorphous portions.

$X_{c}=\frac{I_{c}}{I_{c}+I_{a}} \cdot 100$

where $X_{c}$ - degree of crystallinity [\%], $I_{c}$ - integrated intensity of crystalline region, $I_{a}$ - integrated intensity of amorphous region.

Table 5 shows the degree of crystallinity in TPVs calculated from the WAXD. Upon adding EOC with PP, there is no change in the peak position which suggests that blending only slightly affects the $\alpha$ monoclinic pattern of PP. But peak at

Table 5. Degree of crystallinity $X_{c}$ calculated from WAXD

\begin{tabular}{|l|c|}
\hline \multicolumn{1}{|c|}{ Compound name } & \% Crystallinity \\
\hline PP (Pure) & 50.9 \\
\hline PP-EOC (Unvulcanized) & 49.4 \\
\hline D5 (Dynamically vulcanized) & 46.7 \\
\hline D15 (Dynamically vulcanized) & 29.6 \\
\hline
\end{tabular}




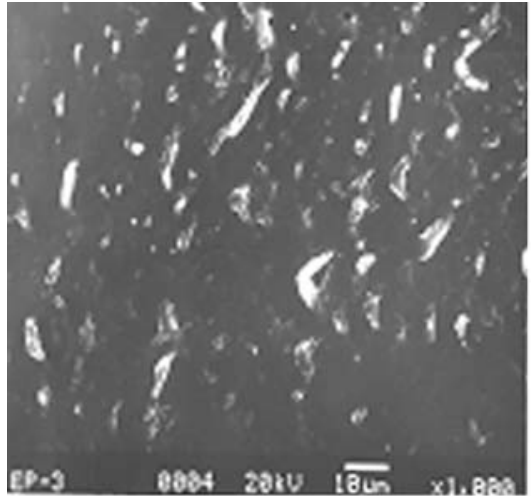

a)

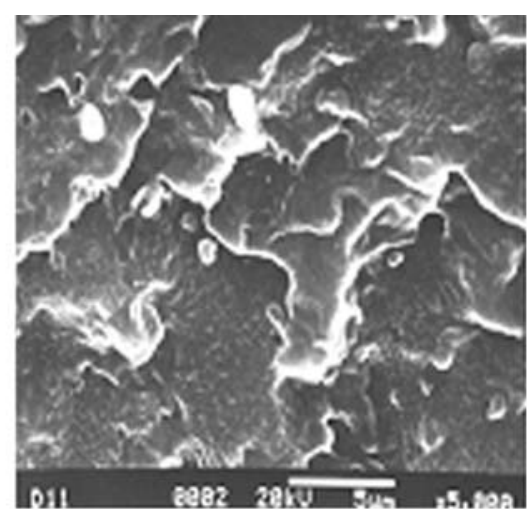

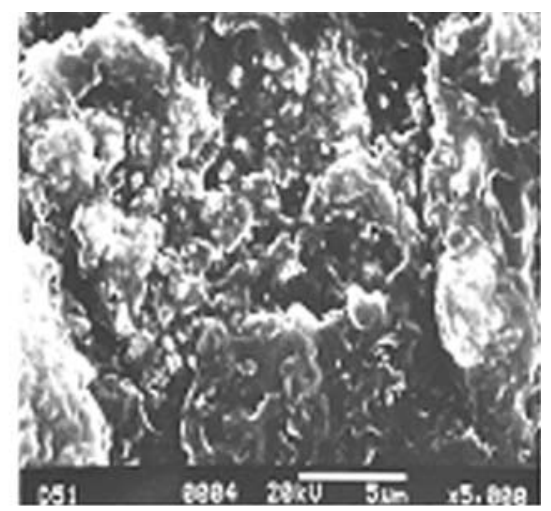

c)

Figure 9. Morphology of the 50/100 PP/EOC blend (a) without dynamic vulcanization (b) 1 meq concentration of DCP (c) 5 meq concentration of DCP

$2 \theta=14.1^{\circ}$ corresponding to $\alpha$ (110) plane intensity decreases indicating a small decrease in crystallinity level. This can be explained by the migration of EOC phase into the interchain spaces of PP. Similar behavior was obtained in PP/EPDM simple blends. It shows that increasing the EPDM content in the blend cause more defective and thinner lamellar crystals [21].

From the WAXS graph (Figure 8) of 5 meq concentration of DCP cured TPVs, again the intensity of the peaks decreases suggest the decrease in the crystallinity value. This may be due to the formation in-situ grafting of EOC with PP, which further reduces the regular arrangement of crystalline region and makes the crystallization process difficult. In order to have a better understanding of the effect of peroxide on the crystalline pattern, $15 \mathrm{meq}$ concentration of DCP cured TPVs was analyzed. As mentioned previously, grafting of EOC on PP and limited extent of crosslinking in PP lead to decrease the crystallinity and the perfectness of the spherulite. Guan et al. [22] also reported that grafting of modifiers (like guanidine, diamide polymers) on PP backbones cause a decrease in crystallinity level and affect the perfectness of spherulites. Variation in the packing in the crystal lattice also inferred from the change in the peak position at 15 meq concentration.

\subsection{Morphology}

Morphology of un-vulcanized and dynamically vulcanized blends of PP/EOC TPVs are shown in Figure 9. In general morphology of un-vulcanized blend shows either a co-continuous phases or larger rubbery particle dispersed in $\mathrm{PP}$ phase or PP particles dispersed in an EOC matrix depending on the blend composition. In this blend system of $\mathrm{PP} / \mathrm{EOC}, \mathrm{EOC}$ being in greater proportion than PP, the former forms continuous phase and PP as dispersed phase. Figure 9a shows the unvulcanized blend of PP/EOC, since both the components having good affinity show the elongated particles of PP dispersed in EOC matrix. During dynamic vulcanization, phase inversion occurs. This is evident because crosslinking of rubbery phase leads to drastic increase in viscosity which forms crosslinked EOC droplets in PP matrix and once it was cross-linked it shows higher stability against coalescence. As these droplets are formed PP becomes the continuous phase which maintains thermoplasticity of the blends. Figures $9 b$ and $9 c$ show the dynamically vulcanized blends with 1 and 5 meq concentration of DCP respectively. There is no much difference in the morphology of different peroxides taken under investigation. However, as the peroxide concentration increases, high degree of vulcanization persist, thereby decreasing the particle size with increasing number of particles [23].

\section{Conclusions}

Novel thermoplastic vulcanizates (TPVs) are prepared by dynamic vulcanization process from 50/100 blend ratio of PP/EOC by coagent assisted peroxide as crosslinking system. Dynamically vulcanized blends show better mechanical and thermal properties than those of unvulcanized blends. Different peroxides show different crosslinking efficiency and different decomposition products hav- 
ing different reactivity. The choice of the best peroxide depends on the extent of crosslinking in EOC phase and also on the extent of degradation in the PP phase. Out of the three peroxides used in this investigation di cumyl peroxide (DCP) shows the best overall balance of properties except tear strength. DTBPIB shows a significant improvement in tear strength. Concentration of peroxide also has significant influence on the mechanical properties. Higher concentrations of peroxides cause increasing brittle failure of the sample due to severe degradation in the PP phase. Peroxide of 3 meq is sufficient to give significant improvement in mechanical properties. From the cure characteristics, different peroxides employed can be ranked on basis of efficiency and reactivity. Crosslinking efficiency can be ranked as DTBPIB $\geq$ DCP $>$ TBCP and cure rate can be ranked as $\mathrm{DCP}>\mathrm{TBCP} \geq$ DTBPIB. Differential scanning calorimetry and morphological analysis show that the blend components are thermodynamically immiscible and exist as a heterogeneous biphasic system. DCP and DTBPIB form relatively highly reactive radicals and hence show better mechanical properties and correspondingly affect more the crystallization of PP. As the concentration of peroxide increases, particle size decreases. However, mechanical properties of these TPVs are not good enough at high concentration, which is due to severe degradation in the PP phase in presence of peroxide. From WAXS data, there is no measurable shift in the peak position suggesting no significant change in the crystallinity pattern with the addition of EOC and also by dynamic vulcanization, but the level of crystallinity (\% crystallinity) is affected by the same.

\section{Acknowledgements}

The authors are thankful to Council for Scientific and Industrial Research (CSIR), New Delhi, India for their financial assistance.

\section{References}

[1] Legge N. R., Holden G., Schroeders H. E.: Thermoplastic elastomer: A comprehensive review. Hanser, Munich (1987).

[2] De S. K., Bhowmick A. K.: Thermoplastic elastomers from rubber-plastic blends. Horwood, London (1990).
[3] Gessler A. M., Haslett W. H.: Process for preparing a vulcanized blend of crystalline polypropylene and chlorinated butyl rubber. US patent 3037954, USA (1962).

[4] Coran A. Y., Patel R.: Rubber-thermoplastic compositions. Part I. EPDM-polypropylene thermoplastic vulcanizates. Rubber Chemistry and Technology, 53, 141-150 (1980).

[5] Abdou-Sabet S., Fath M. A.: Thermoplastic elastomer blends of olefin rubber and polyolefin resin. US patent 4311628, USA (1982).

[6] Loan L. D.: Mechanism of peroxide vulcanization of elastomers. Rubber Chemistry and Technology, 40, 149-176 (1967).

[7] Dluzneski P. R.: Peroxide vulcanization of elastomers. Rubber Chemistry and Technology, 74, 451-490 (2001).

[8] Naskar K., Noordermeer J. W. M.: Dynamically vulcanized PP/EPDM blends-effects of different types of peroxide on the properties. Rubber Chemistry and Technology, 76, 1001-1018 (2003).

[9] Naskar K., Noordermeer J. W. M.: Influence of various peroxides in PP/EPDM thermoplastic vulcanizates at varied blend ratios. Journal of Elastomer and Plastics, 38, 163-180 (2006).

[10] Naskar K., Noordermeer J. W. M.: Dynamically vulcanized PP/EPDM blends-effects: Multifunctional peroxides as crosslinking agents, Part I. Rubber Chemistry and Technology, 77, 955-971 (2004).

[11] Thitithammawong A., Nakason C., Sahakaro K., Noordermeer J. W. M.: Thermoplastic vulcanizate based on epoxidized natural rubber/polypropylene blends, selection of optimal peroxide type and concentration in relation to mixing conditions. European Polymer Journal, 43, 4008-4018 (2007).

[12] Chatterjee K., Naskar K.: Development of thermoplastic elastomers based on maleated ethylene propylene rubber and polypropylene by dynamic vulcanization. Express Polymer Letters, 1, 527-534 (2007).

[13] Karger-Kocsis J.: Thermoplastic rubbers via dynamic vulcanization. in 'Polymer Blends and Alloys', (eds.: Shonaike G. O., Simon G. P.) Marcel Dekker, New York, 125-154 (1999).

[14] Sengupta P.: Morphology of olefinic thermoplastic elastomers. PhD thesis, Twente University, Netherlands (2004).

[15] Naskar K.: Thermoplastic elastomer based on PP/EPDM blends by dynamic vulcanization: A review. Rubber Chemistry and Technology, 80, 504 510 (2007).

[16] Walton K. L.: Metallocene catalysed ethylene/alpha olefin copolymers used in thermoplastic elastomers. Rubber Chemistry and Technology, 77, 552-568 (2004).

[17] George J., Varughese K. T., Thomas S.: Dynamically vulcanized thermoplastic elastomer blends of polyethylene and nitrile rubber. Polymer, 41, 1507-1517 (2000). 
[18] Williams M. G., Harrington B. A., Miller T. M.: New low density EXACT ${ }^{\circledR}$ plastomers as high performance impact modifiers. A technical report by Exxon Mobil Company (2003).

[19] Braton A. F. M.: Handbook of solubility parameters and other cohesion parameters. CRC Press, Boca Raton (1983).

[20] George J., Varughese K. T., Thomas S.: Thermal and crystallisation behavior of isotactic polypropylene/ nitrile rubber blends. Polymer, 41, 5485-5503 (2000).
[21] Bielinski D. M., Slusarski L., Wlochowicz A., Slusarczyk C., Douillard A.: Some aspect of isotactic polypropylene crystallization in an ethylene-propylenediene rubber matrix. Polymer International, 44, 161173 (1997).

[22] Guan Y., Wang S., Zheng A., Xiao H.: Crystallisation behaviour of polypropylene and functional polypropylene. Journal of Applied Polymer Science, 88, 872877 (2003).

[23] Abdou-Sabet S., Patel R. P.: Morphology of elastomeric alloys. Rubber Chemistry and Technology, 64, 769-779 (1991). 